\title{
Evidence for the decay $\mathrm{B}_{\mathrm{c}}^{+} \rightarrow \mathrm{J} / \psi 3 \pi^{+} 2 \pi^{-}$
}

\section{LHCh}

\section{The LHCb collaboration}

\section{E-mail: Ivan.Belyaev@cern.ch}

ABSTRACT: Evidence is presented for the decay $\mathrm{B}_{\mathrm{c}}^{+} \rightarrow \mathrm{J} / \psi 3 \pi^{+} 2 \pi^{-}$using proton-proton collision data, corresponding to an integrated luminosity of $3 \mathrm{fb}^{-1}$, collected with the $\mathrm{LHCb}$ detector. A signal yield of $32 \pm 8$ decays is found with a significance of 4.5 standard deviations. The ratio of the branching fraction of the $\mathrm{B}_{\mathrm{c}}^{+} \rightarrow \mathrm{J} / \psi 3 \pi^{+} 2 \pi^{-}$decay to that of the $\mathrm{B}_{\mathrm{c}}^{+} \rightarrow \mathrm{J} / \psi \pi^{+}$decay is measured to be

$$
\frac{\mathcal{B}\left(\mathrm{B}_{\mathrm{c}}^{+} \rightarrow \mathrm{J} / \psi 3 \pi^{+} 2 \pi^{-}\right)}{\mathcal{B}\left(\mathrm{B}_{\mathrm{c}}^{+} \rightarrow \mathrm{J} / \psi \pi^{+}\right)}=1.74 \pm 0.44 \pm 0.24
$$

where the first uncertainty is statistical and the second is systematic.

KEYwords: Hadron-Hadron Scattering, QCD, Branching fraction, B physics, Flavor physics

ARXIV EPRINT: 1404.0287 


\section{Contents}

1 Introduction 1

2 Detector 2

3 Candidate selection $\quad 2$

4 Signal and normalization yields 3

$5 \quad$ Efficiency and systematic uncertainties 5

6 Results and summary $\quad 8$

The LHCb collaboration $\quad 12$

\section{Introduction}

The $\mathrm{B}_{\mathrm{c}}^{+}$meson is the only meson consisting of two heavy quarks of different flavours. It was discovered by the CDF collaboration through the semileptonic decay $\mathrm{B}_{\mathrm{c}}^{+} \rightarrow \mathrm{J} / \psi \ell^{+} \boldsymbol{v}_{\ell} \mathrm{X}$ [1], where $\mathrm{X}$ denotes possible unobserved particles. ${ }^{1}$ The CDF collaboration also observed the hadronic decay mode $\mathrm{B}_{\mathrm{c}}^{+} \rightarrow \mathrm{J} / \psi \pi^{+}[2]$. Recently, the LHCb experiment has observed several new channels including $\mathrm{B}_{\mathrm{c}}^{+} \rightarrow \mathrm{J} / \psi \pi^{+} \pi^{+} \pi^{-}[3], \mathrm{B}_{\mathrm{c}}^{+} \rightarrow \psi(2 \mathrm{~S}) \pi^{+}[4], \mathrm{B}_{\mathrm{c}}^{+} \rightarrow \mathrm{J} / \psi \mathrm{D}_{\mathrm{s}}^{+}$and $\mathrm{B}_{\mathrm{c}}^{+} \rightarrow \mathrm{J} / \psi \mathrm{D}_{\mathrm{s}}^{*+}[5], \mathrm{B}_{\mathrm{c}}^{+} \rightarrow \mathrm{J} / \psi \mathrm{K}^{+}[6], \mathrm{B}_{\mathrm{c}}^{+} \rightarrow \mathrm{J} / \psi \mathrm{K}^{+} \mathrm{K}^{-} \pi^{+}[7]$ and $\mathrm{B}_{\mathrm{c}}^{+} \rightarrow \mathrm{B}_{\mathrm{s}}^{0} \pi^{+}$[8]. The lifetime of the $\mathrm{B}_{\mathrm{c}}^{+}$meson $[9,10]$ is about three times shorter than that of the $\mathrm{B}^{0}$ and $\mathrm{B}^{+}$ mesons, confirming the important role played by the $\mathrm{c}$ quark in $\mathrm{B}_{\mathrm{c}}^{+}$decays. The decays of $\mathrm{B}_{\mathrm{c}}^{+}$mesons into charmonia and light hadrons are expected to be well described by the factorization approximation $[11,12]$. In this scheme, the $\mathrm{B}_{\mathrm{c}}^{+} \rightarrow \mathrm{J} / \psi 3 \pi^{+} 2 \pi^{-}$decay is characterized by the form factors of the $\mathrm{B}_{\mathrm{c}}^{+} \rightarrow \mathrm{J} / \psi \mathrm{W}^{+}$transition and the spectral functions for the virtual $\mathrm{W}^{+}$boson into light hadrons [13]. The predictions for the ratio of branching fractions

$$
R_{5 \pi} \equiv \frac{\mathcal{B}\left(\mathrm{B}_{\mathrm{c}}^{+} \rightarrow \mathrm{J} / \psi 3 \pi^{+} 2 \pi^{-}\right)}{\mathcal{B}\left(\mathrm{B}_{\mathrm{c}}^{+} \rightarrow \mathrm{J} / \psi \pi^{+}\right)}
$$

are 0.95 and 1.1 [14], using form factor calculations from refs. [15] and [16], respectively.

In this article, the first evidence for the decay $\mathrm{B}_{\mathrm{c}}^{+} \rightarrow \mathrm{J} / \psi 3 \pi^{+} 2 \pi^{-}$and a measurement of $R_{5 \pi}$ are reported. The analysis is based on a data sample of proton-proton ( $\mathrm{pp}$ ) collisions, corresponding to an integrated luminosity of $1 \mathrm{fb}^{-1}$ at a centre-of-mass energy of $7 \mathrm{TeV}$ and $2 \mathrm{fb}^{-1}$ at $8 \mathrm{TeV}$, collected with the LHCb detector.

\footnotetext{
${ }^{1}$ The inclusion of charge conjugate modes is implicit throughout this paper.
} 


\section{Detector}

The LHCb detector [17] is a single-arm forward spectrometer covering the pseudorapidity range $2<\eta<5$, designed for the study of particles containing b or c quarks. The detector includes a high-precision tracking system consisting of a silicon-strip vertex detector surrounding the pp interaction region, a large-area silicon-strip detector located upstream of a dipole magnet with a bending power of about $4 \mathrm{Tm}$, and three stations of silicon-strip detectors and straw drift tubes [18] placed downstream. The combined tracking system provides a momentum measurement with relative uncertainty that varies from $0.4 \%$ at $5 \mathrm{GeV} / c$ to $0.6 \%$ at $100 \mathrm{GeV} / c$, and impact parameter resolution of $20 \mu \mathrm{m}$ for tracks with large transverse momentum. Different types of charged hadrons are distinguished using information from two ring-imaging Cherenkov detectors [19]. Photon, electron and hadron candidates are identified by a calorimeter system consisting of scintillating-pad and preshower detectors, an electromagnetic calorimeter and a hadronic calorimeter. Muons are identified by a system composed of alternating layers of iron and multiwire proportional chambers [20]. The trigger [21] consists of a hardware stage, based on information from the calorimeter and muon systems, followed by a software stage, which applies a full event reconstruction.

This analysis uses events collected by triggers that select the $\mu^{+} \mu^{-}$pair from the $\mathrm{J} / \psi$ decay with high efficiency. At the hardware stage either one or two muon candidates are required to trigger the event. In the case of single muon triggers, the transverse momentum, $p_{\mathrm{T}}$, of the muon candidate is required to be greater than $1.5 \mathrm{GeV} / c$. For dimuon candidates, the product of the $p_{\mathrm{T}}$ of muon candidates is required to satisfy $\sqrt{p_{\mathrm{T} 1} p_{\mathrm{T} 2}}>1.3 \mathrm{GeV} / c$. At the subsequent software trigger stage, two muons are selected with an invariant mass in the range $2.97<m_{\mu^{+} \mu^{-}}<3.21 \mathrm{GeV} / c^{2}$ and consistent with originating from a common vertex. The common vertex is required to be significantly displaced from the pp collision vertices.

Simulated pp collisions are generated using PYTHIA 6.4 [22] with the configuration described in ref. [23]. Final-state QED radiative corrections are included using the Рнотоs package [24]. The $\mathrm{B}_{\mathrm{c}}^{+}$mesons are produced by a dedicated generator, BCVEgPy [25]. The decays of all hadrons are performed by EvtGen [26], and a specific model is implemented to generate the decays $\mathrm{B}_{\mathrm{c}}^{+} \rightarrow \mathrm{J} / \psi 3 \pi^{+} 2 \pi^{-}$, assuming factorization [14]. The model allows the implementation of different form factors for this decay, calculated using QCD sum rules [15] or a relativistic quark model [16]. These predictions lead to very similar values and those based on the relativistic quark model are used in the simulation. The coupling of the five pion $\left(3 \pi^{+} 2 \pi^{-}\right)$system to the virtual $\mathrm{W}^{+}$is taken from $\tau^{+}$lepton decays [27]. The interaction of the generated particles with the detector and its response are implemented using the GEANT4 toolkit [28, 29] as described in ref. [30].

\section{Candidate selection}

The decays $\mathrm{B}_{\mathrm{c}}^{+} \rightarrow \mathrm{J} / \psi 3 \pi^{+} 2 \pi^{-}$and $\mathrm{B}_{\mathrm{c}}^{+} \rightarrow \mathrm{J} / \psi \pi^{+}$are reconstructed using the $\mathrm{J} / \psi \rightarrow \mu^{+} \mu^{-}$decay mode. The selection criteria chosen are similar for both channels. 
All tracks are required to be in the pseudorapidity range $2<\eta<4.9$. Good track quality of charged particles is ensured by requiring the $\chi^{2}$ per number of degrees of freedom, $\chi^{2} /$ ndf, provided by the track fit, to be less than 3 . Suppression of fake tracks created by the reconstruction is achieved by a neural network trained with simulated samples to discriminate between fake tracks and tracks associated with real particles [31], ensuring the rate of fake tracks below $0.3 \%$.

Two dedicated neural networks are used for muon and pion identification. These networks use the information from the Cherenkov detectors [19], muon chambers [32] and the calorimeter system [33], together with the tracking information. The momentum of the pion candidates is required to be between $3.2 \mathrm{GeV} / c$ and $150 \mathrm{GeV} / c$ in order to ensure good quality particle identification in Cherenkov detectors. The requirements on the neural network output are chosen to ensure good agreement between data and simulation and significant reduction of the background due to misidentification.

Pairs of oppositely charged muons, originating from a common vertex, are combined to form $\mathrm{J} / \psi \rightarrow \mu^{+} \mu^{-}$candidates. The $p_{\mathrm{T}}$ of each muon is required to be greater than $550 \mathrm{MeV} / c$. Good vertex reconstruction is ensured by requiring the $\chi^{2}$ of the vertex fit, $\chi_{\mathrm{vtx}}^{2}$, to be less than 20 . To select dimuon vertices that are well-separated from the reconstructed pp interaction vertices, the decay length is required to be at least three times its uncertainty. The invariant mass of the dimuon combination is required to be between 3.020 and $3.135 \mathrm{GeV} / c^{2}$. The asymmetric mass range with respect to the known $\mathrm{J} / \psi$ meson mass [9] is chosen to include the QED radiative tail.

The selected $\mathrm{J} / \psi$ candidates are combined with pions to form $\mathrm{B}_{\mathrm{c}}^{+} \rightarrow \mathrm{J} / \psi 3 \pi^{+} 2 \pi^{-}$and $\mathrm{B}_{\mathrm{c}}^{+} \rightarrow \mathrm{J} / \psi \pi^{+}$candidates. The transverse momentum of each pion is required to be greater than $400 \mathrm{MeV} / c$. To ensure that the pions are inconsistent with being directly produced in a pp interaction, the impact parameter $\chi^{2}$, defined as the difference between the $\chi^{2}$ values of the fits of the pp collision vertex formed with and without the considered pion track, is required to satisfy $\chi_{\mathrm{IP}}^{2}>4$. When more than one primary vertex is reconstructed, the vertex with the smallest value of $\chi_{\mathrm{IP}}^{2}$ is chosen. Good vertex reconstruction for the $\mathrm{B}_{\mathrm{c}}^{+}$candi-

date vertex is ensured by requiring the $\chi_{\mathrm{vtx}}^{2} / \mathrm{ndf}$ to be less than 12 . To suppress the large combinatorial background in the $\mathrm{B}_{\mathrm{c}}^{+} \rightarrow \mathrm{J} / \psi 3 \pi^{+} 2 \pi^{-}$sample, the $\chi^{2}$ of the vertex fit for all $\mathrm{J} / \psi \pi^{ \pm}$combinations, as well as for all dipion combinations, is required to be less than 20. To improve the invariant mass resolution, a kinematic fit [34] is performed that constrains the $\mu^{+} \mu^{-}$pair to the known mass of the $\mathrm{J} / \psi$ meson. It is also required that the $\mathrm{B}_{\mathrm{c}}^{+}$candidate's momentum vector points back to from the associated pp interaction vertex. The $\chi^{2}$ per number of degrees of freedom of the fit, $\chi_{\mathrm{fit}}^{2} / \mathrm{ndf}$, is required to be less than 5. The measured decay time of the $\mathrm{B}_{\mathrm{c}}^{+}$candidate, calculated with respect to the associated primary vertex, is required to be between $150 \mu \mathrm{m} / c$ and $1 \mathrm{~mm} / c$.

\section{Signal and normalization yields}

The mass distribution for the selected $\mathrm{J} / \psi 3 \pi^{+} 2 \pi^{-}$candidates is shown in figure 1 . To estimate the signal yield, an extended maximum likelihood fit to the unbinned mass distribution is made. The $\mathrm{B}_{\mathrm{c}}^{+} \rightarrow \mathrm{J} / \psi 3 \pi^{+} 2 \pi^{-}$signal is modelled by a Gaussian distribution 


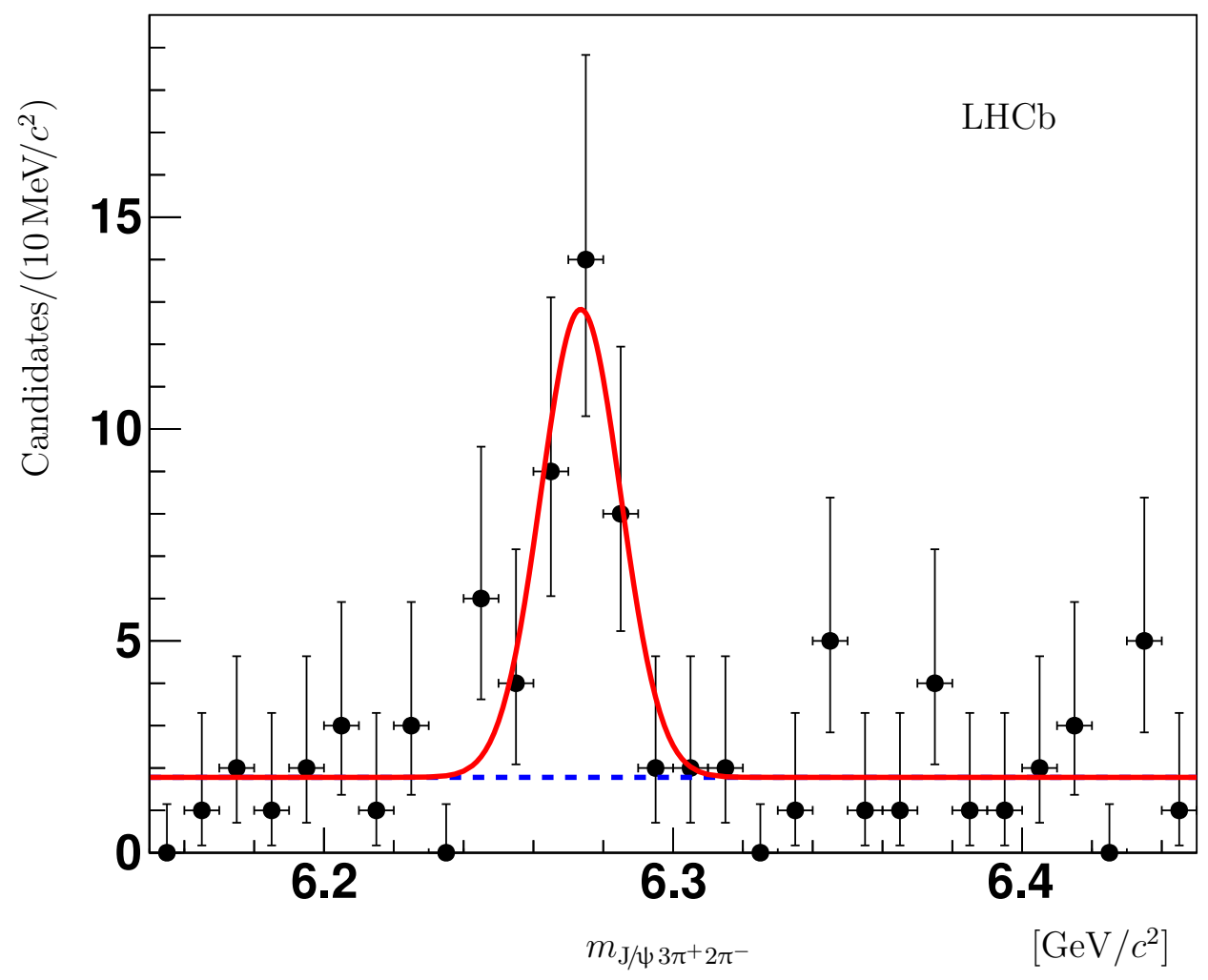

Figure 1. Mass distribution for selected $\mathrm{B}_{\mathrm{c}}^{+} \rightarrow \mathrm{J} / \psi 3 \pi^{+} 2 \pi^{-}$candidates. The result of a fit using the model described in the text (red solid line) is shown together with the background component (blue dashed line).

and the background by a constant function. The fit results for the fitted mass and mass resolution of $\mathrm{B}_{\mathrm{c}}^{+}$signal, $m_{\mathrm{B}_{\mathrm{c}}^{+}}$and $\sigma_{\mathrm{B}_{\mathrm{c}}^{+}}$, and signal yield $N_{\mathrm{B}_{\mathrm{c}}^{+} \rightarrow \mathrm{J} / 43 \pi^{+} 2 \pi^{-}}$, are listed in table 1 ,

The statistical significance for the observed signal is determined as $\mathcal{S}_{\sigma}=\sqrt{-2 \log \frac{\mathcal{L}_{\mathrm{B}}}{\mathcal{L}_{\mathrm{S}+\mathrm{B}}}}$ where $\mathcal{L}_{\mathrm{S}+\mathrm{B}}$ and $\mathcal{L}_{\mathrm{B}}$ denote the likelihood associated with the signal-plus-background and background-only hypothesis, respectively. The likelihoods are calculated with the peak position fixed to the known mass of $\mathrm{B}_{\mathrm{c}}^{+}$meson $[5,9]$ and the mass resolution fixed to $10.1 \mathrm{MeV} / \mathrm{c}^{2}$ as expected from simulation. The statistical significance of the $\mathrm{B}_{\mathrm{c}}^{+} \rightarrow \mathrm{J} / \psi 3 \pi^{+} 2 \pi^{-}$signal is 4.5 standard deviations.

For the selected $\mathrm{B}_{\mathrm{c}}^{+}$candidates, the existence of resonant structures is searched for in the $\pi^{+} \pi^{-}, \pi^{+} \pi^{+} \pi^{-}, \pi^{+} \pi^{-} \pi^{-}, 2 \pi^{+} 2 \pi^{-}, 3 \pi^{+} 2 \pi^{-}$and $\mathrm{J} / \psi \pi^{+} \pi^{-}$combinations of final state particles using the sPlot technique [35], with the reconstructed $\mathrm{J} / \psi 3 \pi^{+} 2 \pi^{-}$mass as discriminating variable, to subtract the background. No significant narrow structures are observed; in particular, no indication of a contribution from $\mathrm{B}_{\mathrm{c}}^{+} \rightarrow \psi(2 \mathrm{~S}) \pi^{+} \pi^{+} \pi^{-}$, followed by the $\psi(2 \mathrm{~S}) \rightarrow \mathrm{J} / \psi \pi^{+} \pi^{-}$decay, is seen. The background-subtracted five-pion mass distribution is shown in figure 2, along with the theoretical prediction in ref. [14], which describes the data well. The consistency between data and the model prediction is estimated using a $\chi^{2}$-test and gives a $p$-value of $14 \%$. The corresponding $p$-value for the phase space decay model is $4 \%$. 


\begin{tabular}{|lcc|}
\hline \multicolumn{2}{|c|}{ Parameter } & Value \\
\hline$m_{\mathrm{B}_{\mathrm{c}}^{+}}$ & {$\left[\mathrm{MeV} / c^{2}\right]$} & $6273 \pm 3$ \\
$\sigma_{\mathrm{B}_{\mathrm{c}}^{+}}$ & {$\left[\mathrm{MeV} / c^{2}\right]$} & $11.4 \pm 3.4$ \\
$N_{\mathrm{B}_{\mathrm{c}}^{+} \rightarrow \mathrm{J} / \psi 3 \pi^{+} 2 \pi^{-}}$ & & $32 \pm 8$ \\
\hline
\end{tabular}

Table 1. Signal parameters of the unbinned extended maximum likelihood fit to the $\mathrm{J} / \psi 3 \pi^{+} 2 \pi^{-}$mass distribution. Uncertainties are statistical only.

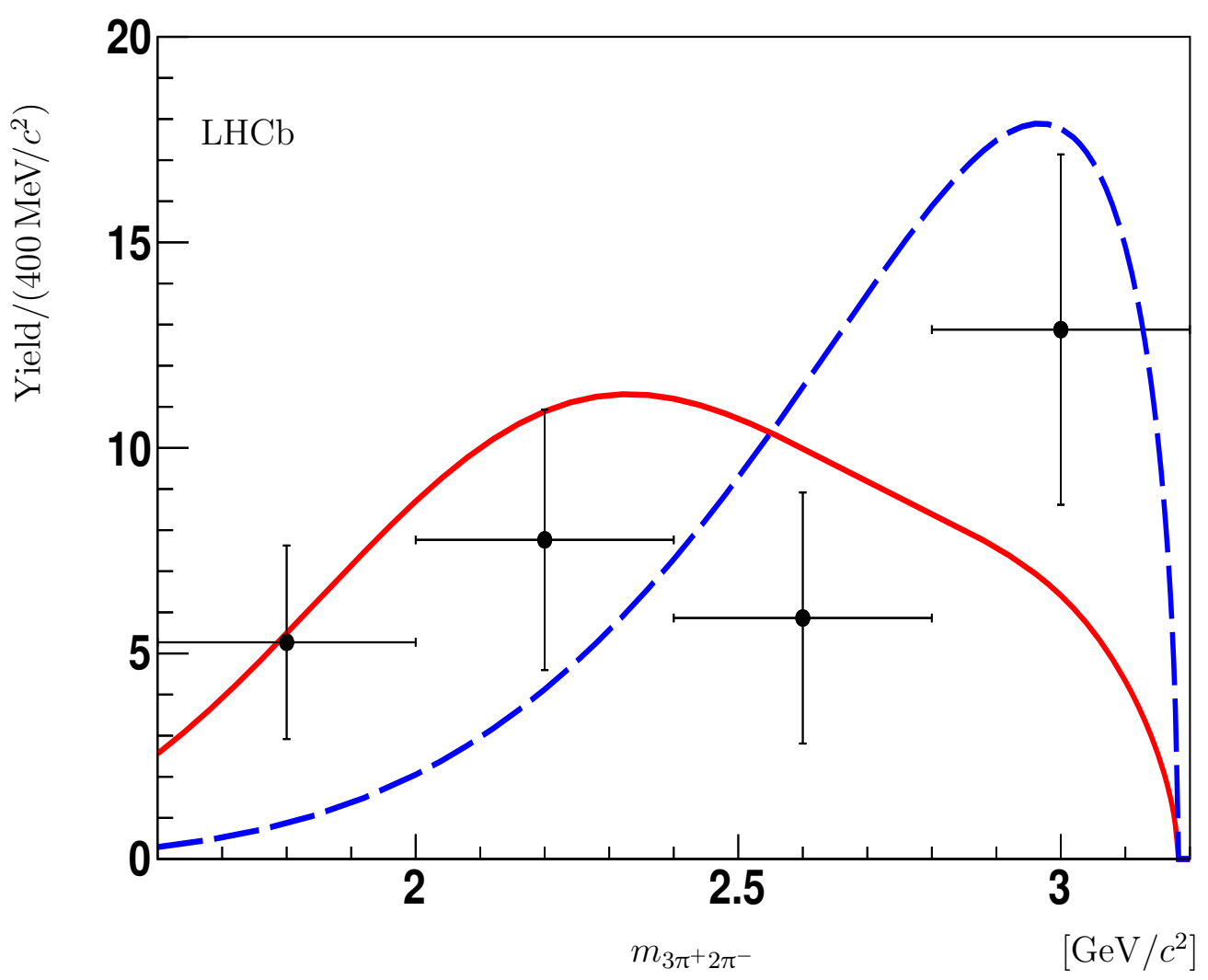

Figure 2. Background-subtracted distribution of five-pion mass from $B_{c}^{+} \rightarrow J / \psi 3 \pi^{+} 2 \pi^{-}$events (points with error bars). The model prediction from ref. [14] is shown by a red solid line, and the expectation from the phase space model is shown by a blue dashed line.

The mass distribution of the selected $\mathrm{B}_{\mathrm{c}}^{+} \rightarrow \mathrm{J} / \psi \pi^{+}$candidates is shown in figure 3, together with the result of an extended unbinned maximum likelihood fit. The $\mathrm{B}_{\mathrm{c}}^{+}$signal is modelled by a Gaussian distribution and the background by an exponential function. The fit gives a yield of $2271 \pm 63$ events.

\section{$5 \quad$ Efficiency and systematic uncertainties}

The overall efficiency for each decay is the product of the geometrical acceptance of the detector, reconstruction, selection and trigger efficiencies. These are estimated using simula- 


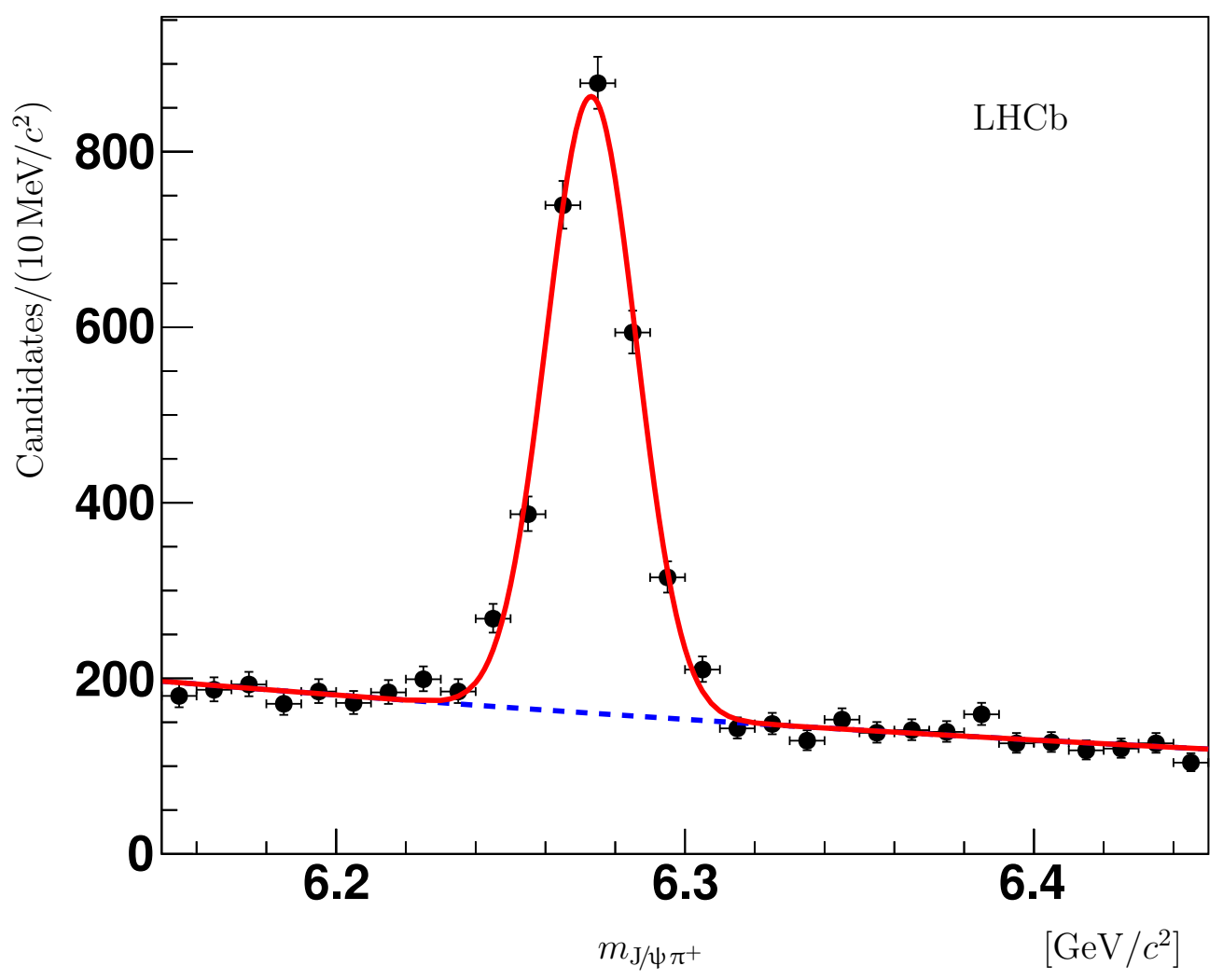

Figure 3. Mass distribution for selected $\mathrm{B}_{\mathrm{c}}^{+} \rightarrow \mathrm{J} / \psi \pi^{+}$candidates. The result of a fit using the model described in the text (red solid line) is shown together with the background component (blue dashed line).

tion and the ratio of the efficiencies is found to be

$$
\frac{\varepsilon\left(\mathrm{B}_{\mathrm{c}}^{+} \rightarrow \mathrm{J} / \psi \pi^{+}\right)}{\varepsilon\left(\mathrm{B}_{\mathrm{c}}^{+} \rightarrow \mathrm{J} / \psi 3 \pi^{+} 2 \pi^{-}\right)}=123.8 \pm 5.6 \pm 15.1
$$

where the first uncertainty is statistical, due to the finite size of the simulated sample, and the second one is systematic, as discussed below. The large difference in efficiencies is due to the reconstruction of four additional low- $p_{\mathrm{T}}$ pions in the $\mathrm{B}_{\mathrm{c}}^{+} \rightarrow \mathrm{J} / \psi 3 \pi^{+} 2 \pi^{-}$mode. The efficiencies for the data samples collected at a centre-of-mass energy of $7 \mathrm{TeV}$ and $8 \mathrm{TeV}$ are found to be similar and a luminosity-weighted average is used, with the corresponding systematic uncertainty discussed below.

Many sources of systematic uncertainty cancel in the ratio, in particular those related to the muon and $\mathrm{J} / \Psi$ reconstruction and identification. Those that do not cancel are discussed below and summarized in table 2 .

A systematic uncertainty arises from the imperfect knowledge of the shape of the signal and background in the $\mathrm{J} / \psi 3 \pi^{+} 2 \pi^{-}$and $\mathrm{J} / \psi \pi^{+}$mass distributions. The dependence of the signal yields on the fit model is studied by varying the signal and background parameterizations. This is assessed by using Crystal Ball [36] and double-sided Crystal Ball [37] functions for the parameterization of the $\mathrm{B}_{\mathrm{c}}^{+}$signals. The background parametrization 


\begin{tabular}{|lc|}
\hline Source & Uncertainty [\%] \\
\hline Fit model & 6.6 \\
Decay model & \\
$m_{3 \pi^{+}+\pi^{-}}$reweighting & 7.7 \\
$\Psi(2 \mathrm{~S})$ mass veto & 3.1 \\
Data-simulation agreement & \\
Hadron interactions & $4 \times 2.0$ \\
Track quality selection & $4 \times 0.6$ \\
Trigger & 1.1 \\
Pion identification & 0.7 \\
Selection variables & 1.0 \\
B lifetime & 0.9 \\
Stability for various data taking conditions & 2.5 \\
Acceptance & 0.8 \\
\hline Total & 13.9 \\
\hline
\end{tabular}

Table 2. Relative systematic uncertainties for the ratio $R_{5 \pi}$. The total uncertainty is the quadratic sum of the individual components.

is performed using both exponential and polynomial functions. The maximum observed change of $6.6 \%$ in the ratio of $\mathrm{B}_{\mathrm{c}}^{+} \rightarrow \mathrm{J} / \psi 3 \pi^{+} 2 \pi^{-}$and $\mathrm{B}_{\mathrm{c}}^{+} \rightarrow \mathrm{J} / \psi \pi^{+}$yields is assigned as a systematic uncertainty.

To assess the systematic uncertainty related to the $\mathrm{B}_{\mathrm{c}}^{+} \rightarrow \mathrm{J} / \psi 3 \pi^{+} 2 \pi^{-}$decay model used in the simulation [14], the reconstructed mass distribution of the five-pion system in simulated events is reweighted to reproduce the distribution observed in data. As a cross-check the efficiency is also recalculated using a phase space model for the $\mathrm{B}_{\mathrm{c}}^{+} \rightarrow \mathrm{J} / \psi 3 \pi^{+} 2 \pi^{-}$decays. There is a maximal change in efficiency of $7.7 \%$, which is taken as the systematic uncertainty for the decay model. In addition, the analysis is repeated with the removal of all $\mathrm{B}_{\mathrm{c}}^{+}$candidates where the $\mathrm{J} / \psi \pi^{+} \pi^{-}$mass is compatible with originating from $\psi(2 \mathrm{~S}) \rightarrow \mathrm{J} / \psi \pi^{+} \pi^{-}$decays. The observed difference of $3.1 \%$ is assigned as an additional systematic uncertainty.

A large class of uncertainties arises from the differences between data and simulation, in particular those affecting the efficiency for reconstruction of charged-particle tracks. The largest of these arises from the simulation of hadronic interactions in the detector, which has an uncertainty of $2 \%$ per track $[31,38,39]$. An additional uncertainty associated with the track quality requirements for the additional four pions in the signal decay is estimated to be $0.6 \%$ per track $[5,7]$. The trigger efficiency for events with $\mathrm{J} / \psi \rightarrow \mu^{+} \mu^{-}$produced in beauty hadron decays is studied on data in high-yield modes [5, 40] and a systematic uncertainty of $1.1 \%$ is assigned based on the comparison of the ratio of trigger efficiencies for high-yield samples of $\mathrm{B}^{+} \rightarrow \mathrm{J} / \psi \mathrm{K}^{+}$and $\mathrm{B}^{+} \rightarrow \psi(2 \mathrm{~S}) \mathrm{K}^{+}$decays on data and simulation [40]. 
The systematic uncertainty associated with pion identification is studied using a sample of $\mathrm{B}^{+} \rightarrow \mathrm{J} / \psi \mathrm{K}^{+} \pi^{+} \pi^{-}$decays. The efficiency to identify a $\pi^{+} \pi^{-}$pair is compared for data and simulation. This comparison shows a $0.35 \%$ difference between the data and simulation in the efficiency to identify a pion pair. As a result of this study an uncertainty of $0.7 \%$ is assigned for the four additional pions in the analysis.

The transverse momentum and rapidity spectra for the selected $\mathrm{B}_{\mathrm{c}}^{+} \rightarrow \mathrm{J} / \psi \pi^{+}$candidates, as well their daughter $\mathrm{J} / \psi$ mesons and pions, are found to be in good agreement with the predictions from the BCVEGPY generator. Good agreement in efficiencies determined from the data and simulation has been observed for all variables used in the selection of $\mathrm{B}_{\mathrm{c}}^{+} \rightarrow \mathrm{J} / \psi \pi^{+}$candidates. The differences do not exceed $1 \%$, which is used as a conservative estimate for the systematic uncertainty from the selection variables. The agreement between data and simulation has also been cross-checked using the $\mathrm{B}_{\mathrm{c}}^{+} \rightarrow \mathrm{J} / \psi 3 \pi^{+} 2 \pi^{-}$signal by varying the selection criteria to the values that correspond to a $20 \%$ change in the signal yield in simulation. No unexpectedly large deviation is found.

The different acceptance as a function of decay time for the $\mathrm{B}_{\mathrm{c}}^{+} \rightarrow \mathrm{J} / \psi 3 \pi^{+} 2 \pi^{-}$and $\mathrm{B}_{\mathrm{c}}^{+} \rightarrow \mathrm{J} / \psi \pi^{+}$decay modes results in an additional systematic uncertainty related to the imprecise knowledge of the $\mathrm{B}_{\mathrm{c}}^{+}$lifetime. To assess the related uncertainty, the decay time distributions for simulated events are reweighted after changing the $\mathrm{B}_{\mathrm{c}}^{+}$lifetime by one standard deviation around the value of $509 \pm 8 \pm 12 \mathrm{fs}$ [10] measured by LHCb and the efficiencies are recomputed. The observed $0.9 \%$ variation in the ratio of efficiencies is used as the systematic uncertainty.

The uncertainty related to the stability of the analysis results against variations of the detector and trigger configurations occuring in different data-taking periods are tested by studying the ratio of the yields of $\mathrm{B}^{+} \rightarrow \mathrm{J} / \psi \mathrm{K}^{+} \pi^{+} \pi^{-}$and $\mathrm{B}^{+} \rightarrow \mathrm{J} / \psi \mathrm{K}^{+}$decays as a function of the data-taking period. According to this study an additional systematic uncertainty of $2.5 \%$ is assigned [5].

The last systematic uncertainty originates from the dependence of the geometrical acceptance on both the beam crossing angle and the position of the luminosity region. The resulting $0.8 \%$ difference in the efficiency ratios is taken as an estimate of the systematic uncertainty.

A summary of systematic uncertainties is presented in table 2. The total systematic uncertainty on the ratio of the branching fractions $R_{5 \pi}$ is $13.9 \%$.

\section{Results and summary}

The first evidence for the decay $\mathrm{B}_{\mathrm{c}}^{+} \rightarrow \mathrm{J} / \psi 3 \pi^{+} 2 \pi^{-}$is found using pp collisions, corresponding to an integrated luminosity of $3 \mathrm{fb}^{-1}$, collected with the LHCb detector A signal yield of $32 \pm 8$ events is found. The significance, taking into account the systematic uncertainties due to the fit function, peak position and mass resolution in the fit, is estimated to be 4.5 standard deviations.

Using the $\mathrm{B}_{\mathrm{c}}^{+} \rightarrow \mathrm{J} / \psi \pi^{+}$mode as a normalization channel, the ratio of branching fractions is calculated as

$$
R_{5 \pi}=\frac{N\left(\mathrm{~B}_{\mathrm{c}}^{+} \rightarrow \mathrm{J} / \psi 3 \pi^{+} 2 \pi^{-}\right)}{N\left(\mathrm{~B}_{\mathrm{c}}^{+} \rightarrow \mathrm{J} / \psi \pi^{+}\right)} \times \frac{\varepsilon\left(\mathrm{B}_{\mathrm{c}}^{+} \rightarrow \mathrm{J} / \psi \pi^{+}\right)}{\varepsilon\left(\mathrm{B}_{\mathrm{c}}^{+} \rightarrow \mathrm{J} / \psi 3 \pi^{+} 2 \pi^{-}\right)},
$$


where $N$ is the number of reconstructed decays obtained from the fit described in section 4 and the efficiency ratio is taken from eq. (5.1). The ratio of branching fractions is measured to be

$$
\frac{\mathcal{B}\left(\mathrm{B}_{\mathrm{c}}^{+} \rightarrow \mathrm{J} / \psi 3 \pi^{+} 2 \pi^{-}\right)}{\mathcal{B}\left(\mathrm{B}_{\mathrm{c}}^{+} \rightarrow \mathrm{J} / \psi \pi^{+}\right)}=1.74 \pm 0.44 \pm 0.24,
$$

where the first uncertainty is statistical and the second is systematic. The result is in agreement with theoretical predictions [14] of 0.95 and 1.1 using the form factors from refs. [15] and [16], respectively. This result is also consistent with analogous measurements in $\mathrm{B}^{0}$ and $\mathrm{B}^{+}$meson decays [9]

$$
\begin{aligned}
& \frac{\mathcal{B}\left(\mathrm{B}^{0} \rightarrow \mathrm{D}^{*-} 3 \pi^{+} 2 \pi^{-}\right)}{\mathcal{B}\left(\mathrm{B}^{0} \rightarrow \mathrm{D}^{*-} \pi^{+}\right)}=1.70 \pm 0.34, \\
& \frac{\mathcal{B}\left(\mathrm{B}^{+} \rightarrow \overline{\mathrm{D}}^{* 0} 3 \pi^{+} 2 \pi^{-}\right)}{\mathcal{B}\left(\mathrm{B}^{+} \rightarrow \overline{\mathrm{D}}^{* 0} \pi^{+}\right)}=1.10 \pm 0.24,
\end{aligned}
$$

as expected from factorization.

\section{Acknowledgments}

We thank A.K. Likhoded and A.V. Luchinky for fruitful discussions about the dynamics of $\mathrm{B}_{\mathrm{c}}^{+}$decays. We express our gratitude to our colleagues in the CERN accelerator departments for the excellent performance of the LHC. We thank the technical and administrative staff at the LHCb institutes. We acknowledge support from CERN and from the national agencies: CAPES, CNPq, FAPERJ and FINEP (Brazil); NSFC (China); CNRS/IN2P3 and Region Auvergne (France); BMBF, DFG, HGF and MPG (Germany); SFI (Ireland); INFN (Italy); FOM and NWO (The Netherlands); SCSR (Poland); MEN/IFA (Romania); MinES, Rosatom, RFBR and NRC "Kurchatov Institute" (Russia); MinECo, XuntaGal and GENCAT (Spain); SNSF and SER (Switzerland); NASU (Ukraine); STFC and the Royal Society (United Kingdom); NSF (U.S.A.). We also acknowledge the support received from EPLANET, Marie Curie Actions and the ERC under FP7. The Tier1 computing centres are supported by IN2P3 (France), KIT and BMBF (Germany), INFN (Italy), NWO and SURF (The Netherlands), PIC (Spain), GridPP (United Kingdom). We are indebted to the communities behind the multiple open source software packages on which we depend. We are also thankful for the computing resources and the access to software R\&D tools provided by Yandex LLC (Russia).

Open Access. This article is distributed under the terms of the Creative Commons Attribution License (CC-BY 4.0), which permits any use, distribution and reproduction in any medium, provided the original author(s) and source are credited.

\section{References}

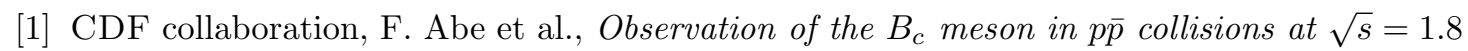
TeV, Phys. Rev. Lett. 81 (1998) 2432 [hep-ex/9805034] [INSPIRE]. 
[2] CDF collaboration, T. Aaltonen et al., Observation of the Decay $B_{c}^{+} \rightarrow J / \psi \pi^{ \pm}$and Measurement of the $B_{c}^{+}$Mass, Phys. Rev. Lett. 100 (2008) 182002 [arXiv:0712.1506] [INSPIRE].

[3] LHCb collaboration, First observation of the decay $B_{c}^{+} \rightarrow J / \psi \pi^{+} \pi^{-} \pi^{+}$, Phys. Rev. Lett. 108 (2012) 251802 [arXiv:1204.0079] [INSPIRE].

[4] LHCb collaboration, Observation of the decay $B_{c}^{+} \rightarrow \psi(2 S) \pi^{+}$, Phys. Rev. D 87 (2013) 071103 [arXiv: 1303.1737] [INSPIRE].

[5] LHCb collaboration, Observation of $B_{c}^{+} \rightarrow J / \psi D_{s}^{+}$and $B_{c}^{+} \rightarrow J / \psi D_{s}^{*+}$ decays, Phys. Rev. D 87 (2013) 112012 [arXiv: 1304.4530] [INSPIRE].

[6] LHCb collaboration, First observation of the decay $B_{c}^{+} \rightarrow J / \psi K^{+}$, JHEP 09 (2013) 075 [arXiv: 1306.6723] [INSPIRE].

[7] LHCb collaboration, Observation of the decay $B_{c}^{+} \rightarrow J / \psi K^{+} K^{-} \pi^{+}$, JHEP 11 (2013) 094 [arXiv: 1309.0587] [INSPIRE].

[8] LHCb collaboration, Observation of the decay $B_{c}^{+} \rightarrow B_{s}^{0} \pi^{+}$, Phys. Rev. Lett. 111 (2013) 181801 [arXiv: 1308.4544] [INSPIRE].

[9] Particle Data Group collaboration, J. Beringer et al., Review of particle physics, Phys. Rev. D 86 (2012) 010001 [INSPIRE].

[10] LHCb collaboration, Measurement of the $B_{c}^{+}$meson lifetime using $B_{c}^{+} \rightarrow J / \psi \mu^{+} \nu_{\mu} X$ decays, arXiv: 1401.6932 [INSPIRE].

[11] M. Bauer, B. Stech and M. Wirbel, Exclusive nonleptonic decays of $D-, D_{s^{-}}$and B-mesons, Z. Phys. C 34 (1987) 103 [inSPIRE].

[12] M. Wirbel, Description of weak decays of D and B Mesons, Prog. Part. Nucl. Phys. 21 (1988) 33 [INSPIRE].

[13] A. Likhoded and A. Luchinsky, Light hadron production in $B_{c} \rightarrow J / \psi+X$ decays, Phys. Rev. D 81 (2010) 014015 [arXiv:0910.3089] [INSPIRE].

[14] A. Luchinsky, Production of charged $\pi$-mesons in exclusive $B_{c} \rightarrow V(P)+n \pi$ decays, Phys. Rev. D 86 (2012) 074024 [arXiv:1208.1398] [INSPIRE].

[15] V. Kiselev, A. Kovalsky and A. Likhoded, $B_{c}$ decays and lifetime in QCD sum rules, Nucl. Phys. B 585 (2000) 353 [hep-ph/0002127] [INSPIRE].

[16] D. Ebert, R. Faustov and V. Galkin, Weak decays of the $B_{c}$ meson to charmonium and D mesons in the relativistic quark model, Phys. Rev. D 68 (2003) 094020 [hep-ph/0306306] [INSPIRE].

[17] LHCb collaboration, The LHCb Detector at the LHC, 2008 JINST 3 S08005 [INSPIRE].

[18] R. Arink et al., Performance of the LHCb Outer Tracker, 2014 JINST 901002 [arXiv: 1311.3893] [INSPIRE].

[19] M. Adinolfi et al., Performance of the LHCb RICH detector at the LHC, Eur. Phys. J. C 73 (2013) 2431 [arXiv: 1211.6759] [INSPIRE].

[20] J. Alves et al., Performance of the LHCb muon system, 2013 JINST 8 P02022 [arXiv: 1211.1346] [INSPIRE].

[21] R. Aaij et al., The LHCb trigger and its performance in 2011, 2013 JINST 8 P04022 [arXiv:1211.3055] [INSPIRE]. 
[22] T. Sjöstrand, S. Mrenna and P.Z. Skands, PYTHIA 6.4 physics and manual, JHEP 05 (2006) 026 [hep-ph/0603175] [INSPIRE].

[23] I. Belyaev et al., Handling of the generation of primary events in Gauss, the LHCb simulation framework, IEEE Nucl. Sci. Symp. Conf. Rec. IEEE (2010) 1155.

[24] P. Golonka and Z. Was, PHOTOS Monte Carlo: a precision tool for QED corrections in Z and $W$ decays, Eur. Phys. J. C 45 (2006) 97 [hep-ph/0506026] [INSPIRE].

[25] C.-H. Chang, C. Driouichi, P. Eerola and X.G. Wu, BCVEGPY: an event generator for hadronic production of the $B_{c}$ meson, Comput. Phys. Commun. 159 (2004) 192 [hep-ph/0309120] [INSPIRE].

[26] D. Lange, The EvtGen particle decay simulation package, Nucl. Instrum. Meth. A 462 (2001) 152 [INSPIRE].

[27] BABAR collaboration, J. Lees et al., Study of high-multiplicity 3-prong and 5-prong $\tau$ decays at BABAR, Phys. Rev. D 86 (2012) 092010 [arXiv: 1209.2734] [INSPIRE].

[28] GeAnt4 collaboration, J. Allison et al., GEANT4 developments and applications, IEEE Trans. Nucl. Sci. 53 (2006) 270.

[29] GEANT4 collaboration, S. Agostinelli et al., GEANT4 - a simulation toolkit, Nucl. Instrum. Meth. A 506 (2003) 250 [INSPIRE].

[30] M. Clemencic et al., The LHCb simulation application, Gauss: design, evolution and experience, J. Phys. Conf. Ser. 331 (2011) 032023 [inSPIRE].

[31] R. Aaij et al., Measurement of the track reconstruction efficiency at LHCb, LHCb-DP-2013-002, in preparation.

[32] F. Archilli et al., Performance of the muon identification at LHCb, 2013 JINST 8 P10020 [arXiv: 1306.0249] [INSPIRE].

[33] R. Aaij et al., Performance of the LHCb calorimeters, LHCb-DP-2013-004, in preparation.

[34] W.D. Hulsbergen, Decay chain fitting with a Kalman filter, Nucl. Instrum. Meth. A 552 (2005) 566 [physics/0503191] [INSPIRE].

[35] M. Pivk and F.R. Le Diberder, SPlot: a statistical tool to unfold data distributions, Nucl. Instrum. Meth. A 555 (2005) 356 [physics/0402083] [INSPIRE].

[36] T. Skwarnicki, A study of the radiative cascade transitions between the $\Upsilon^{\prime}$ and $\Upsilon$ resonances, Ph.D. thesis, Institute of Nuclear Physics, Krakow, Poland (1986), DESY-F31-86-02 [INSPIRE].

[37] LHCb collaboration, Observation of $J / \psi$ pair production in pp collisions at $\sqrt{s}=7 \mathrm{TeV}$, Phys. Lett. B 707 (2012) 52 [arXiv:1109.0963] [INSPIRE].

[38] A. Jaeger et al., Measurement of the track finding efficiency, LHCb-PUB-2011-025 (2011).

[39] LHCb collaboration, Prompt $K_{s}^{0}$ production in pp collisions at $\sqrt{s}=0.9 \mathrm{TeV}$, Phys. Lett. $\mathbf{B}$ 693 (2010) 69 [arXiv: 1008.3105] [INSPIRE].

[40] LHCb collaboration, Measurement of relative branching fractions of $B$ decays to $\psi(2 S)$ and

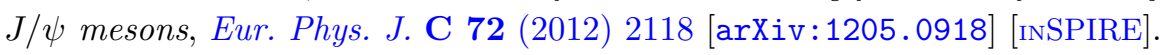




\section{The LHCb collaboration}

R. Aaij ${ }^{41}$, B. Adeva ${ }^{37}$, M. Adinolfi ${ }^{46}$, A. Affolder ${ }^{52}$, Z. Ajaltouni ${ }^{5}$, J. Albrecht ${ }^{9}$, F. Alessio ${ }^{38}$, M. Alexander ${ }^{51}$, S. Ali ${ }^{41}$, G. Alkhazov ${ }^{30}$, P. Alvarez Cartelle ${ }^{37}$, A.A. Alves Jr ${ }^{25,38}$, S. Amato ${ }^{2}$, S. Amerio ${ }^{22}$, Y. Amhis ${ }^{7}$, L. An ${ }^{3}$, L. Anderlini ${ }^{17, g}$, J. Anderson ${ }^{40}$, R. Andreassen ${ }^{57}$, M. Andreotti ${ }^{16, f}$, J.E. Andrews ${ }^{58}$, R.B. Appleby ${ }^{54}$, O. Aquines Gutierrez ${ }^{10}$, F. Archilli ${ }^{38}$, A. Artamonov ${ }^{35}$, M. Artuso ${ }^{59}$, E. Aslanides ${ }^{6}$, G. Auriemma ${ }^{25, m}$, M. Baalouch ${ }^{5}$, S. Bachmann $^{11}$, J.J. Back ${ }^{48}$, A. Badalov ${ }^{36}$, V. Balagura ${ }^{31}$, W. Baldini ${ }^{16}$, R.J. Barlow ${ }^{54}$, C. Barschel ${ }^{38}$, S. Barsuk ${ }^{7}$, W. Barter ${ }^{47}$, V. Batozskaya ${ }^{28}$, Th. Bauer ${ }^{41}$, A. Bay ${ }^{39}$, J. Beddow ${ }^{51}$, F. Bedeschi ${ }^{23}$, I. Bediaga ${ }^{1}$, S. Belogurov ${ }^{31}$, K. Belous ${ }^{35}$, I. Belyaev ${ }^{31}$, E. Ben-Haim ${ }^{8}$, G. Bencivenni ${ }^{18}$, S. Benson ${ }^{50}$, J. Benton ${ }^{46}$, A. Berezhnoy ${ }^{32}$, R. Bernet ${ }^{40}$, M.-O. Bettler ${ }^{47}$, M. van Beuzekom ${ }^{41}$, A. Bien ${ }^{11}$, S. Bifani ${ }^{45}$, T. Bird ${ }^{54}$, A. Bizzeti ${ }^{17, i}$, P.M. Bjørnstad ${ }^{54}$, T. Blake ${ }^{48}$, F. Blanc $^{39}$, J. Blouw ${ }^{10}$, S. Blusk ${ }^{59}$, V. Bocci ${ }^{25}$, A. Bondar ${ }^{34}$, N. Bondar ${ }^{30,38}$, W. Bonivento ${ }^{15,38}$, S. Borghi ${ }^{54}$, A. Borgia ${ }^{59}$, M. Borsato ${ }^{7}$, T.J.V. Bowcock ${ }^{52}$, E. Bowen ${ }^{40}$, C. Bozzi ${ }^{16}$, T. Brambach ${ }^{9}$, J. van den Brand ${ }^{42}$, J. Bressieux ${ }^{39}$, D. Brett ${ }^{54}$, M. Britsch ${ }^{10}$, T. Britton ${ }^{59}$, N.H. Brook ${ }^{46}$, H. Brown ${ }^{52}$, A. Bursche ${ }^{40}$, G. Busetto ${ }^{22, p}$, J. Buytaert ${ }^{38}$, S. Cadeddu ${ }^{15}$, R. Calabrese ${ }^{16, f}$, O. Callot ${ }^{7}$, M. Calvi ${ }^{20, k}$, M. Calvo Gomez ${ }^{36, n}$, A. Camboni ${ }^{36}$, P. Campana ${ }^{18,38}$, D. Campora Perez ${ }^{38}$, F. Caponio ${ }^{21, t}$, A. Carbone ${ }^{14, d}$, G. Carboni ${ }^{24, l}$, R. Cardinale ${ }^{19,38, j}$, A. Cardini ${ }^{15}$, H. Carranza-Mejia ${ }^{50}$, L. Carson ${ }^{50}$, K. Carvalho Akiba ${ }^{2}$, G. Casse $^{52}$, L. Cassina ${ }^{20}$, L. Castillo Garcia ${ }^{38}$, M. Cattaneo ${ }^{38}$, Ch. Cauet $^{9}$, R. Cenci ${ }^{58}$, M. Charles ${ }^{8}$, Ph. Charpentier ${ }^{38}$, S.-F. Cheung ${ }^{55}$, N. Chiapolini ${ }^{40}$, M. Chrzaszcz ${ }^{40,26}$, K. Ciba ${ }^{38}$, X. Cid Vidal ${ }^{38}$, G. Ciezarek ${ }^{53}$, P.E.L. Clarke ${ }^{50}$, M. Clemencic ${ }^{38}$, H.V. Cliff ${ }^{47}$, J. Closier ${ }^{38}$, C. Coca ${ }^{29}$, V. Coco $^{38}$, J. Cogan ${ }^{6}$, E. Cogneras ${ }^{5}$, P. Collins ${ }^{38}$, A. Comerma-Montells ${ }^{11}$, A. Contu ${ }^{15,38}$, A. Cook $^{46}$, M. Coombes ${ }^{46}$, S. Coquereau ${ }^{8}$, G. Corti ${ }^{38}$, I. Counts ${ }^{56}$, B. Couturier ${ }^{38}$, G.A. Cowan ${ }^{50}$, D.C. Craik ${ }^{48}$, M. Cruz Torres ${ }^{60}$, S. Cunliffe ${ }^{53}$, R. Currie ${ }^{50}$, C. D'Ambrosio ${ }^{38}$, J. Dalseno ${ }^{46}$, P. David ${ }^{8}$, P.N.Y. David ${ }^{41}$, A. Davis ${ }^{57}$, K. De Bruyn ${ }^{41}$, S. De Capua ${ }^{54}$, M. De Cian $^{11}$, J.M. De Miranda ${ }^{1}$, L. De Paula ${ }^{2}$, W. De Silva ${ }^{57}$, P. De Simone ${ }^{18}$, D. Decamp ${ }^{4}$, M. Deckenhoff ${ }^{9}$, L. Del Buono ${ }^{8}$, N. Déléage ${ }^{4}$, D. Derkach ${ }^{55}$, O. Deschamps ${ }^{5}$, F. Dettori ${ }^{42}$, A. Di Canto ${ }^{38}$, H. Dijkstra ${ }^{38}$, S. Donleavy ${ }^{52}$, F. Dordei ${ }^{11}$, M. Dorigo ${ }^{39}$, A. Dosil Suárez ${ }^{37}$, D. Dossett ${ }^{48}$, A. Dovbnya ${ }^{43}$, F. Dupertuis ${ }^{39}$, P. Durante ${ }^{38}$, R. Dzhelyadin ${ }^{35}$, A. Dziurda ${ }^{26}$, A. Dzyuba ${ }^{30}$, S. Easo ${ }^{49}$, U. Egede ${ }^{53}$, V. Egorychev ${ }^{31}$, S. Eidelman ${ }^{34}$, S. Eisenhardt ${ }^{50}$, U. Eitschberger ${ }^{9}$, R. Ekelhof ${ }^{9}$, L. Eklund ${ }^{51,38}$, I. El Rifai ${ }^{5}$, Ch. Elsasser ${ }^{40}$, S. Esen ${ }^{11}$, A. Falabella ${ }^{16, f}$, C. Färber ${ }^{11}$, C. Farinelli ${ }^{41}$, N. Farley ${ }^{45}$, S. Farry ${ }^{52}$, D. Ferguson ${ }^{50}$, V. Fernandez Albor ${ }^{37}$, F. Ferreira Rodrigues ${ }^{1}$, M. Ferro-Luzzi ${ }^{38}$, S. Filippov ${ }^{33}$, M. Fiore ${ }^{16, f}$, M. Fiorini ${ }^{16, f}$, M. Firlej ${ }^{27}$, C. Fitzpatrick ${ }^{38}$, T. Fiutowski ${ }^{27}$, M. Fontana ${ }^{10}$, F. Fontanelli ${ }^{19, j}$, R. Forty ${ }^{38}$, O. Francisco ${ }^{2}$, M. Frank $^{38}$, C. Frei ${ }^{38}$, M. Frosini ${ }^{17,38, g}$, J. Fu ${ }^{21}$, E. Furfaro ${ }^{24, l}$, A. Gallas Torreira ${ }^{37}$, D. Galli ${ }^{14, d}$, S. Gallorini ${ }^{22}$, S. Gambetta ${ }^{19, j}$, M. Gandelman ${ }^{2}$, P. Gandini ${ }^{59}$, Y. Gao ${ }^{3}$, J. Garofoli ${ }^{59}$, J. Garra Tico ${ }^{47}$, L. Garrido ${ }^{36}$, C. Gaspar ${ }^{38}$, R. Gauld ${ }^{55}$,

L. Gavardi ${ }^{9}$, E. Gersabeck ${ }^{11}$, M. Gersabeck ${ }^{54}$, T. Gershon ${ }^{48}$, Ph. Ghez $^{4}$, A. Gianelle ${ }^{22}$,

S. Giani ${ }^{39}$, V. Gibson ${ }^{47}$, L. Giubega ${ }^{29}$, V.V. Gligorov ${ }^{38}$, C. Göbel ${ }^{60}$, D. Golubkov ${ }^{31}$,

A. Golutvin ${ }^{53,31,38}$, A. Gomes ${ }^{1, a}$, H. Gordon ${ }^{38}$, C. Gotti ${ }^{20}$, M. Grabalosa Gándara ${ }^{5}$,

R. Graciani Diaz ${ }^{36}$, L.A. Granado Cardoso ${ }^{38}$, E. Graugés ${ }^{36}$, G. Graziani ${ }^{17}$, A. Grecu ${ }^{29}$, E. Greening ${ }^{55}$, S. Gregson ${ }^{47}$, P. Griffith ${ }^{45}$, L. Grillo ${ }^{11}$, O. Grünberg ${ }^{62}$, B. Gui ${ }^{59}$, E. Gushchin ${ }^{33}$, Yu. Guz ${ }^{35,38}$, T. Gys ${ }^{38}$, C. Hadjivasiliou ${ }^{59}$, G. Haefeli ${ }^{39}$, C. Haen ${ }^{38}$, T.W. Hafkenscheid ${ }^{65}$, S.C. Haines ${ }^{47}$, S. Hall ${ }^{53}$, B. Hamilton ${ }^{58}$, T. Hampson ${ }^{46}$, X. Han ${ }^{11}$, S. Hansmann-Menzemer ${ }^{11}$,

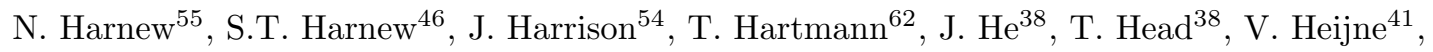
K. Hennessy ${ }^{52}$, P. Henrard ${ }^{5}$, L. Henry ${ }^{8}$, J.A. Hernando Morata ${ }^{37}$, E. van Herwijnen ${ }^{38}$, M. Heß ${ }^{62}$, A. Hicheur ${ }^{1}$, D. Hill ${ }^{55}$, M. Hoballah ${ }^{5}$, C. Hombach ${ }^{54}$, W. Hulsbergen ${ }^{41}$, P. Hunt ${ }^{55}$, N. Hussain ${ }^{55}$, D. Hutchcroft ${ }^{52}$, D. Hynds ${ }^{51}$, M. Idzik ${ }^{27}$, P. Ilten ${ }^{56}$, R. Jacobsson ${ }^{38}$, A. Jaeger ${ }^{11}$, E. Jans ${ }^{41}$, 
P. Jaton ${ }^{39}$, A. Jawahery ${ }^{58}$, M. Jezabek ${ }^{26}$, F. Jing ${ }^{3}$, M. John ${ }^{55}$, D. Johnson ${ }^{55}$, C.R. Jones ${ }^{47}$, C. Joram ${ }^{38}$, B. Jost ${ }^{38}$, N. Jurik ${ }^{59}$, M. Kaballo ${ }^{9}$, S. Kandybei ${ }^{43}$, W. Kanso ${ }^{6}$, M. Karacson ${ }^{38}$, T.M. Karbach ${ }^{38}$, M. Kelsey ${ }^{59}$, I.R. Kenyon ${ }^{45}$, T. Ketel ${ }^{42}$, B. Khanji ${ }^{20}$, C. Khurewathanakul ${ }^{39}$, S. Klaver ${ }^{54}$, O. Kochebina ${ }^{7}$, M. Kolpin ${ }^{11}$, I. Komarov ${ }^{39}$, R.F. Koopman ${ }^{42}$, P. Koppenburg ${ }^{41,38}$, M. Korolev ${ }^{32}$, A. Kozlinskiy ${ }^{41}$, L. Kravchuk ${ }^{33}$, K. Kreplin ${ }^{11}$, M. Kreps ${ }^{48}$, G. Krocker ${ }^{11}$, P. Krokovny ${ }^{34}$, F. Kruse ${ }^{9}$, M. Kucharczyk ${ }^{20,26,38, k}$, V. Kudryavtsev ${ }^{34}$, K. Kurek ${ }^{28}$, T. Kvaratskheliya ${ }^{31}$, V.N. La Thi ${ }^{39}$, D. Lacarrere ${ }^{38}$, G. Lafferty ${ }^{54}$, A. Lai ${ }^{15}$, D. Lambert ${ }^{50}$, R.W. Lambert ${ }^{42}$, E. Lanciotti ${ }^{38}$, G. Lanfranchi ${ }^{18}$, C. Langenbruch ${ }^{38}$, B. Langhans ${ }^{38}$, T. Latham ${ }^{48}$, C. Lazzeroni ${ }^{45}$, R. Le $\mathrm{Gac}^{6}$, J. van Leerdam ${ }^{41}$, J.-P. Lees ${ }^{4}$, R. Lefèvre ${ }^{5}$, A. Leflat ${ }^{32}$,

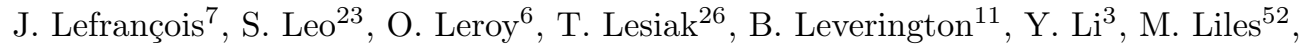

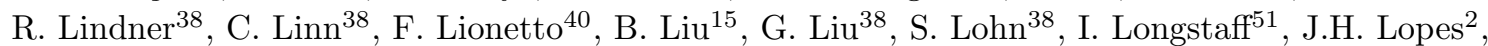
N. Lopez-March ${ }^{39}$, P. Lowdon ${ }^{40}$, H. Lu ${ }^{3}$, D. Lucchesi ${ }^{22, p}, \mathrm{H}_{\text {L Luo }}{ }^{50}$, E. Luppi ${ }^{16, f}$, O. Lupton ${ }^{55}$,

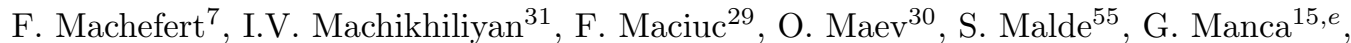
G. Mancinelli ${ }^{6}$, M. Manzali ${ }^{16, f}$, J. Maratas ${ }^{5}$, J.F. Marchand ${ }^{4}$, U. Marconi ${ }^{14}$, C. Marin Benito ${ }^{36}$, P. Marino ${ }^{23, r}$, R. Märki ${ }^{39}$, J. Marks ${ }^{11}$, G. Martellotti ${ }^{25}$, A. Martens ${ }^{8}$, A. Martín Sánchez ${ }^{7}$, M. Martinelli ${ }^{41}$, D. Martinez Santos ${ }^{42}$, F. Martinez Vidal ${ }^{64}$, D. Martins Tostes ${ }^{2}$, A. Massafferri ${ }^{1}$, R. Matev ${ }^{38}$, Z. Mathe ${ }^{38}$, C. Matteuzzi ${ }^{20}$, A. Mazurov ${ }^{16, f}$, M. McCann ${ }^{53}$, J. McCarthy ${ }^{45}$, A. $\mathrm{McNab}^{54}$, R. McNulty ${ }^{12}$, B. McSkelly ${ }^{52}$, B. Meadows ${ }^{57,55}$, F. Meier ${ }^{9}$, M. Meissner ${ }^{11}$, M. Merk ${ }^{41}$, D.A. Milanes ${ }^{8}$, M.-N. Minard ${ }^{4}$, J. Molina Rodriguez ${ }^{60}$, S. Monteil ${ }^{5}$, D. Moran ${ }^{54}$, M. Morandin ${ }^{22}$, P. Morawski ${ }^{26}$, A. Mordà ${ }^{6}$, M.J. Morello ${ }^{23, r}$, J. Moron ${ }^{27}$, R. Mountain ${ }^{59}$, F. Muheim ${ }^{50}$, K. Müller ${ }^{40}$, R. Muresan ${ }^{29}$, B. Muster ${ }^{39}$, P. Naik ${ }^{46}$, T. Nakada ${ }^{39}$, R. Nandakumar ${ }^{49}$, I. Nasteva ${ }^{2}$, M. Needham ${ }^{50}$, N. Neri ${ }^{21}$, S. Neubert ${ }^{38}$, N. Neufeld ${ }^{38}$, M. Neuner ${ }^{11}$, A.D. Nguyen ${ }^{39}$, T.D. Nguyen ${ }^{39}$, C. Nguyen-Mau ${ }^{39, o}$, M. Nicol ${ }^{7}$, V. Niess ${ }^{5}$, R. Niet $^{9}$, N. Nikitin ${ }^{32}$, T. Nikodem ${ }^{11}$, A. Novoselov ${ }^{35}$, A. Oblakowska-Mucha ${ }^{27}$, V. Obraztsov ${ }^{35}$, S. Oggero ${ }^{41}$, S. Ogilvy ${ }^{51}$, O. Okhrimenko ${ }^{44}$, R. Oldeman ${ }^{15, e}$, G. Onderwater ${ }^{65}$, M. Orlandea $^{29}$, J.M. Otalora Goicochea ${ }^{2}$, P. Owen ${ }^{53}$, A. Oyanguren ${ }^{36}$, B.K. Pal ${ }^{59}$, A. Palano ${ }^{13, c}$, F. Palombo ${ }^{21, s}$, M. Palutan ${ }^{18}$, J. Panman ${ }^{38}$, A. Papanestis ${ }^{49,38}$, M. Pappagallo ${ }^{51}$, L. Pappalardo ${ }^{16}$, C. Parkes ${ }^{54}$, C.J. Parkinson ${ }^{9}$, G. Passaleva ${ }^{17}$, G.D. Patel ${ }^{52}$, M. Patel ${ }^{53}$, C. Patrignani ${ }^{19, j}$, A. Pazos Alvarez ${ }^{37}$, A. Pearce ${ }^{54}$, A. Pellegrino ${ }^{41}$, M. Pepe Altarelli ${ }^{38}$, S. Perazzini ${ }^{14, d}$, E. Perez Trigo ${ }^{37}$, P. Perret ${ }^{5}$, M. Perrin-Terrin ${ }^{6}$, L. Pescatore ${ }^{45}$, E. Pesen ${ }^{66}$, K. Petridis ${ }^{53}$, A. Petrolini ${ }^{19, j}$, E. Picatoste Olloqui ${ }^{36}$, B. Pietrzyk ${ }^{4}$, T. Pilař ${ }^{48}$, D. Pinci ${ }^{25}$, A. Pistone ${ }^{19}$, S. Playfer ${ }^{50}$, M. Plo Casasus ${ }^{37}$, F. Polci ${ }^{8}$, A. Poluektov ${ }^{48,34}$, E. Polycarpo ${ }^{2}$, A. Popov ${ }^{35}$, D. Popov ${ }^{10}$, B. Popovici ${ }^{29}$, C. Potterat ${ }^{2}$, A. Powell ${ }^{55}$, J. Prisciandaro ${ }^{39}$, A. Pritchard ${ }^{52}$, C. Prouve ${ }^{46}$, V. Pugatch ${ }^{44}$, A. Puig Navarro ${ }^{39}$, G. Punzi ${ }^{23, q}$, W. Qian ${ }^{4}$, B. Rachwal ${ }^{26}$, J.H. Rademacker ${ }^{46}$, B. Rakotomiaramanana ${ }^{39}$, M. Rama ${ }^{18}$, M.S. Rangel ${ }^{2}$, I. Raniuk ${ }^{43}$, N. Rauschmayr ${ }^{38}$, G. Raven ${ }^{42}$, S. Reichert ${ }^{54}$, M.M. Reid ${ }^{48}$, A.C. dos Reis ${ }^{1}$, S. Ricciardi ${ }^{49}$, A. Richards ${ }^{53}$, K. Rinnert ${ }^{52}$, V. Rives Molina ${ }^{36}$, D.A. Roa Romero ${ }^{5}$, P. Robbe ${ }^{7}$, D.A. Roberts ${ }^{58}$, A.B. Rodrigues ${ }^{1}$, E. Rodrigues ${ }^{54}$, P. Rodriguez Perez ${ }^{54}$, S. Roiser ${ }^{38}$, V. Romanovsky ${ }^{35}$, A. Romero Vidal ${ }^{37}$, M. Rotondo ${ }^{22}$, J. Rouvinet ${ }^{39}$, T. Ruf ${ }^{38}$, F. Ruffini ${ }^{23}$, H. Ruiz ${ }^{36}$, P. Ruiz Valls ${ }^{36}$, G. Sabatino ${ }^{25, l}$, J.J. Saborido Silva ${ }^{37}$, N. Sagidova ${ }^{30}$, P. Sail ${ }^{51}$, B. Saitta ${ }^{15, e}$, V. Salustino Guimaraes ${ }^{2}$,

B. Sanmartin Sedes ${ }^{37}$, R. Santacesaria ${ }^{25}$, C. Santamarina Rios ${ }^{37}$, E. Santovetti ${ }^{24, l}$, M. Sapunov ${ }^{6}$, A. Sarti ${ }^{18}$, C. Satriano ${ }^{25, m}$, A. Satta ${ }^{24}$, M. Savrie ${ }^{16, f}$, D. Savrina ${ }^{31,32}$, M. Schiller ${ }^{42}$, H. Schindler ${ }^{38}$, M. Schlupp ${ }^{9}$, M. Schmelling ${ }^{10}$, B. Schmidt ${ }^{38}$, O. Schneider ${ }^{39}$, A. Schopper ${ }^{38}$, M.-H. Schune ${ }^{7}$, R. Schwemmer ${ }^{38}$, B. Sciascia ${ }^{18}$, A. Sciubba ${ }^{25}$, M. Seco ${ }^{37}$, A. Semennikov ${ }^{31}$, K. Senderowska ${ }^{27}$, I. Sepp ${ }^{53}$, N. Serra ${ }^{40}$, J. Serrano ${ }^{6}$, P. Seyfert ${ }^{11}$, M. Shapkin ${ }^{35}$, I. Shapoval ${ }^{16,43, f}$, Y. Shcheglov ${ }^{30}$, T. Shears ${ }^{52}$, L. Shekhtman ${ }^{34}$, O. Shevchenko ${ }^{43}$, V. Shevchenko ${ }^{63}$, A. Shires ${ }^{9}$, F. Sidorov ${ }^{31}$, R. Silva Coutinho ${ }^{48}$, G. Simi ${ }^{22}$, M. Sirendi ${ }^{47}$,

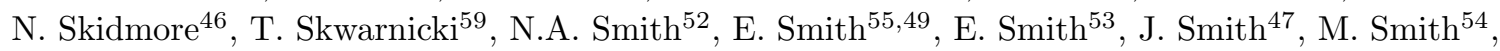


H. Snoek ${ }^{41}$, M.D. Sokoloff ${ }^{57}$, F.J.P. Soler ${ }^{51}$, F. Soomro ${ }^{39}$, D. Souza ${ }^{46}$, B. Souza De Paula ${ }^{2}$, B. $\operatorname{Spaan}^{9}$, A. Sparkes ${ }^{50}$, F. Spinella ${ }^{23}$, P. Spradlin ${ }^{51}$, F. Stagni ${ }^{38}$, S. Stahl ${ }^{11}$, O. Steinkamp ${ }^{40}$, S. Stevenson ${ }^{55}$, S. Stoica ${ }^{29}$, S. Stone ${ }^{59}$, B. Storaci ${ }^{40}$, S. Stracka ${ }^{23,38}$, M. Straticiuc ${ }^{29}$, U. Straumann ${ }^{40}$, R. Stroili ${ }^{22}$, V.K. Subbiah ${ }^{38}$, L. Sun ${ }^{57}$, W. Sutcliffe ${ }^{53}$, K. Swientek ${ }^{27}$, S. Swientek ${ }^{9}$, V. Syropoulos ${ }^{42}$, M. Szczekowski ${ }^{28}$, P. Szczypka ${ }^{39,38}$, D. Szilard ${ }^{2}$, T. Szumlak ${ }^{27}$,

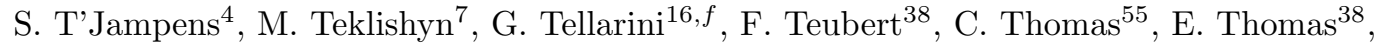
J. van Tilburg ${ }^{41}$, V. Tisserand ${ }^{4}$, M. Tobin ${ }^{39}$, S. Tolk ${ }^{42}$, L. Tomassetti ${ }^{16, f}$, D. Tonelli ${ }^{38}$, S. Topp-Joergensen ${ }^{55}$, N. Torr ${ }^{55}$, E. Tournefier ${ }^{4}$, S. Tourneur ${ }^{39}$, M.T. $\operatorname{Tran}^{39}$, M. Tresch ${ }^{40}$,

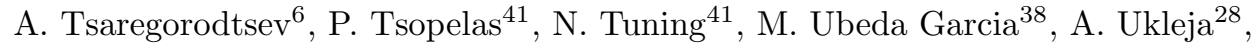

A. Ustyuzhanin ${ }^{63}$, U. Uwer ${ }^{11}$, V. Vagnoni ${ }^{14}$, G. Valenti ${ }^{14}$, A. Vallier ${ }^{7}$, R. Vazquez Gomez ${ }^{18}$, P. Vazquez Regueiro ${ }^{37}$, C. Vázquez Sierra ${ }^{37}$, S. Vecchi ${ }^{16}$, J.J. Velthuis ${ }^{46}$, M. Veltri ${ }^{17, h}$, G. Veneziano ${ }^{39}$, M. Vesterinen ${ }^{11}$, B. Viaud ${ }^{7}$, D. Vieira ${ }^{2}$, X. Vilasis-Cardona ${ }^{36, n}$, A. Vollhardt ${ }^{40}$, D. Volyanskyy ${ }^{10}$, D. Voong ${ }^{46}$, A. $V_{\text {orobyev }}^{30}$, V. Vorobyev ${ }^{34}$, C. Voß ${ }^{62}$, H. Voss ${ }^{10}$, J.A. de Vries ${ }^{41}$, R. Waldi ${ }^{62}$, C. Wallace ${ }^{48}$, R. Wallace ${ }^{12}$, S. Wandernoth ${ }^{11}$, J. Wang ${ }^{59}$, D.R. Ward ${ }^{47}$,

N.K. Watson ${ }^{45}$, D. Websdale ${ }^{53}$, M. Whitehead ${ }^{48}$, J. Wicht ${ }^{38}$, D. Wiedner ${ }^{11}$, G. Wilkinson ${ }^{55}$, M.P. Williams ${ }^{48,49}$, M. Williams ${ }^{56}$, F.F. Wilson ${ }^{49}$, J. Wimberley ${ }^{58}$, J. Wishahi ${ }^{9}$, W. Wislicki ${ }^{28}$, M. Witek ${ }^{26}$, G. Wormser ${ }^{7}$, S.A. Wotton ${ }^{47}$, S. Wright ${ }^{47}$, S. Wu ${ }^{3}, \mathrm{~K}_{\text {. Wyllie }}{ }^{38}, \mathrm{Y} . \mathrm{Xie}^{61}, \mathrm{Z} \mathrm{Xing}^{59}$, Z. Yang ${ }^{3}$, X. Yuan ${ }^{3}$, O. Yushchenko ${ }^{35}$, M. Zangoli ${ }^{14}$, M. Zavertyaev ${ }^{10, b}$, F. Zhang ${ }^{3}$, L. Zhang ${ }^{59}$, W.C. Zhang ${ }^{12}$, Y. Zhang ${ }^{3}$, A. Zhelezov ${ }^{11}$, A. Zhokhov ${ }^{31}$, L. Zhong ${ }^{3}$, A. Zvyagin ${ }^{38}$

1 Centro Brasileiro de Pesquisas Físicas (CBPF), Rio de Janeiro, Brazil

2 Universidade Federal do Rio de Janeiro (UFRJ), Rio de Janeiro, Brazil

3 Center for High Energy Physics, Tsinghua University, Beijing, China

4 LAPP, Université de Savoie, CNRS/IN2P3, Annecy-Le-Vieux, France

5 Clermont Université, Université Blaise Pascal, CNRS/IN2P3, LPC, Clermont-Ferrand, France

6 CPPM, Aix-Marseille Université, CNRS/IN2P3, Marseille, France

7 LAL, Université Paris-Sud, CNRS/IN2P3, Orsay, France

8 LPNHE, Université Pierre et Marie Curie, Université Paris Diderot, CNRS/IN2P3, Paris, France

9 Fakultät Physik, Technische Universität Dortmund, Dortmund, Germany

10 Max-Planck-Institut für Kernphysik (MPIK), Heidelberg, Germany

11 Physikalisches Institut, Ruprecht-Karls-Universität Heidelberg, Heidelberg, Germany

12 School of Physics, University College Dublin, Dublin, Ireland

13 Sezione INFN di Bari, Bari, Italy

14 Sezione INFN di Bologna, Bologna, Italy

15 Sezione INFN di Cagliari, Cagliari, Italy

16 Sezione INFN di Ferrara, Ferrara, Italy

17 Sezione INFN di Firenze, Firenze, Italy

18 Laboratori Nazionali dell'INFN di Frascati, Frascati, Italy

19 Sezione INFN di Genova, Genova, Italy

20 Sezione INFN di Milano Bicocca, Milano, Italy

21 Sezione INFN di Milano, Milano, Italy

22 Sezione INFN di Padova, Padova, Italy

23 Sezione INFN di Pisa, Pisa, Italy

24 Sezione INFN di Roma Tor Vergata, Roma, Italy

25 Sezione INFN di Roma La Sapienza, Roma, Italy

${ }^{26}$ Henryk Niewodniczanski Institute of Nuclear Physics Polish Academy of Sciences, Kraków, Poland

27 AGH - University of Science and Technology, Faculty of Physics and Applied Computer Science, Kraków, Poland

28 National Center for Nuclear Research (NCBJ), Warsaw, Poland

29 Horia Hulubei National Institute of Physics and Nuclear Engineering, Bucharest-Magurele, Romania 
30 Petersburg Nuclear Physics Institute (PNPI), Gatchina, Russia

31 Institute of Theoretical and Experimental Physics (ITEP), Moscow, Russia

32 Institute of Nuclear Physics, Moscow State University (SINP MSU), Moscow, Russia

33 Institute for Nuclear Research of the Russian Academy of Sciences (INR RAN), Moscow, Russia

${ }^{34}$ Budker Institute of Nuclear Physics (SB RAS) and Novosibirsk State University, Novosibirsk, Russia

35 Institute for High Energy Physics (IHEP), Protvino, Russia

36 Universitat de Barcelona, Barcelona, Spain

37 Universidad de Santiago de Compostela, Santiago de Compostela, Spain

38 European Organization for Nuclear Research (CERN), Geneva, Switzerland

39 Ecole Polytechnique Fédérale de Lausanne (EPFL), Lausanne, Switzerland

40 Physik-Institut, Universität Zürich, Zürich, Switzerland

41 Nikhef National Institute for Subatomic Physics, Amsterdam, The Netherlands

42 Nikhef National Institute for Subatomic Physics and VU University Amsterdam, Amsterdam, The Netherlands

43 NSC Kharkiv Institute of Physics and Technology (NSC KIPT), Kharkiv, Ukraine

44 Institute for Nuclear Research of the National Academy of Sciences (KINR), Kyiv, Ukraine

45 University of Birmingham, Birmingham, United Kingdom

46 H.H. Wills Physics Laboratory, University of Bristol, Bristol, United Kingdom

47 Cavendish Laboratory, University of Cambridge, Cambridge, United Kingdom

48 Department of Physics, University of Warwick, Coventry, United Kingdom

49 STFC Rutherford Appleton Laboratory, Didcot, United Kingdom

50 School of Physics and Astronomy, University of Edinburgh, Edinburgh, United Kingdom

51 School of Physics and Astronomy, University of Glasgow, Glasgow, United Kingdom

52 Oliver Lodge Laboratory, University of Liverpool, Liverpool, United Kingdom

53 Imperial College London, London, United Kingdom

54 School of Physics and Astronomy, University of Manchester, Manchester, United Kingdom

55 Department of Physics, University of Oxford, Oxford, United Kingdom

56 Massachusetts Institute of Technology, Cambridge, MA, United States

57 University of Cincinnati, Cincinnati, OH, United States

58 University of Maryland, College Park, MD, United States

59 Syracuse University, Syracuse, NY, United States

60 Pontifícia Universidade Católica do Rio de Janeiro (PUC-Rio), Rio de Janeiro, Brazil, associated to ${ }^{2}$

61 Institute of Particle Physics, Central China Normal University, Wuhan, Hubei, China, associated to ${ }^{3}$

62 Institut für Physik, Universität Rostock, Rostock, Germany, associated to ${ }^{11}$

${ }^{63}$ National Research Centre Kurchatov Institute, Moscow, Russia, associated to ${ }^{31}$

64 Instituto de Fisica Corpuscular (IFIC), Universitat de Valencia-CSIC, Valencia, Spain, associated to ${ }^{36}$

65 KVI - University of Groningen, Groningen, The Netherlands, associated to ${ }^{41}$

${ }^{66}$ Celal Bayar University, Manisa, Turkey, associated to ${ }^{38}$

a Universidade Federal do Triângulo Mineiro (UFTM), Uberaba-MG, Brazil

${ }^{b}$ P.N. Lebedev Physical Institute, Russian Academy of Science (LPI RAS), Moscow, Russia

c Università di Bari, Bari, Italy

d Università di Bologna, Bologna, Italy

e Università di Cagliari, Cagliari, Italy

$f$ Università di Ferrara, Ferrara, Italy

$g$ Università di Firenze, Firenze, Italy

${ }^{h}$ Università di Urbino, Urbino, Italy

${ }^{i}$ Università di Modena e Reggio Emilia, Modena, Italy 
j Università di Genova, Genova, Italy

* Università di Milano Bicocca, Milano, Italy

${ }^{l}$ Università di Roma Tor Vergata, Roma, Italy

$m$ Università della Basilicata, Potenza, Italy

$n$ LIFAELS, La Salle, Universitat Ramon Llull, Barcelona, Spain

- Hanoi University of Science, Hanoi, Viet Nam

p Università di Padova, Padova, Italy

$q$ Università di Pisa, Pisa, Italy

$r$ Scuola Normale Superiore, Pisa, Italy

$s$ Università degli Studi di Milano, Milano, Italy

${ }^{t}$ Politecnico di Milano, Milano, Italy 